Article

\title{
Stereoselective synthesis of vic-halohydrins and an unusual Knoevenagel product from an organocatalyzed aldol reaction: A non-enamine mode
}

\author{
P. B. Thorat, S. V. Goswami, V. P. Sondankar, S. R. Bhusare* \\ Department of Chemistry, Dnyanopasak College, Parbhani-431401, MS, India
}

A R T I C L E I N F O

Article history:

Received 5 January 2015

Accepted 10 February 2015

Published 20 July 2015

\section{Keywords:}

Aldol reaction

Asymmetric synthesis

Chloroacetone

Diastereoselectivity

Hydroxy propanoate

Knoevenagel reaction

Pyrrolidine derivative

\section{A B S T R A C T}

Stereoselective synthesis by an aldol reaction between chloroacetone and aldehyde was studied using a synthesized chiral organocatalyst and triethylamine. The reaction gave $\alpha$-chloro- $\beta$-hydroxy ketones in excellent yield with high anti selectivity and enantioselectivity. The chiral organocatalyst was also used in the Knoevenagel reaction, which gave $\alpha$-cyano- $\beta$-hydroxy ketones at a low temperature and the usual Knoevenagel product at a high temperature. Both products were obtained in good to moderate yield with good anti selectivity in the case of $\alpha$-cyano- $\beta$-hydroxy ketone derivatives.

(C) 2015, Dalian Institute of Chemical Physics, Chinese Academy of Sciences. Published by Elsevier B.V. All rights reserved.

\section{Introduction}

Vic-halohydrins are important intermediates for the synthesis of biologically active compounds and natural products such as bagonists [1], substituted pyrrolidines [2], polychlorosulfolipid [3], and insect sex pheromones [4]. These molecules are building blocks for various bioactive products and natural compounds. Thus efforts have been made to develop effective techniques to synthesize these compounds. The ring opening of enantiomerically pure epoxides is one of the most popular process [5] but suffers from the disadvantage of the formation of regioisomers. The asymmetric reduction of prochiral $\alpha$-halo ketones by chiral catalysts such as oxazaborolidine with borane $[1-2,6], \mathrm{Ru}[1,7]$, or $\mathrm{Rh}[7,8]$ by asymmetric hydrogenation has shown good enantioselectivity.

The condensation reaction of carbonyl compounds with an active methylene compound in the presence of a base is known as Knoevenagel condensation [8]. The Knoevenagel reaction is an important $\mathrm{C}-\mathrm{C}$ bond forming reaction because the synthesized alkenes are very useful intermediates in organic synthesis [9]. For example, Knoevenagel condensation has been successfully combined with hetero-Diels-Alder reactions, Michael addition reactions, ene reactions, and sigmatropic rearrangement for the synthesis of highly functionalized molecules [10-13].

Asymmetric organocatalysis has become an important area of research in organic synthesis. The development of organocatalyzed reactions for the stereoselective construction of $\mathrm{C}-\mathrm{C}$ bonds has been intensively investigated. Since the discovery by List et al. [14] that L-proline can mimic enantioselectively catalyzed intermolecular aldol reactions, many organocatalysts have been synthesized with the aim of increasing their activity and stereoselectivity [15-17]. These reactions are typical of

* Corresponding author. Tel: +91-2452-242411; Fax: +91-2452-242493; E-mail: bhusare71@yahoo.com 
enamine based organocatalysis and proceeds via the reversible condensation of the catalytic amine with a ketone to provide a nucleophilic enamine intermediate. In these reactions, the carboxylic acid functionality on proline is important, and it is postulated that it activates and orients the aldehyde acceptor via a hydrogen bonding interaction.

Alternatively, the utility of a $\mathrm{N}$-sulfinyl group [18-22] as both a chiral directing group and acidifying element in hydrogen-bonded organocatalysts has been demonstrated. In these reactions, the sulfinyl $\mathrm{N}-\mathrm{H}$ bond is postulated to activate the substrate by the formation of a key hydrogen bonding interaction. The inductive electron-withdrawing effect of the sulfinyl group acidifies the $\mathrm{N}-\mathrm{H}$ bond, which serves to modulate the hydrogen bonding interaction. In addition, the close proximity of the stereogenic sulfur to the active site of the catalyst contributes to high stereoselectivity control in these reactions. On the basis of the success of hydrogen bonding organocatalysis, we studied pyrrolidine based organocatalysis in the absence of a Lewis basic primary or secondary amine functionality, which occurs exclusively via hydrogen bonding and van der Waals interactions.

We restricted enamine/imine formation by introducing an acetyl group onto the active nitrogen site in the pyrrolidine ring and replaced the carboxylic group by an amide and sulfinyl group. To perform hydrogen bonding organocatalysis, we synthesized the organocatalysts shown in Fig. 1. Earlier results have been reported in a previous communication [23].

\section{Experimental}

\subsection{General details}

All solvents used were commercial anhydrous grade used without further purification. Aluminium sheets $20 \mathrm{~cm} \times 20 \mathrm{~cm}$, silica gel $60 \mathrm{~F}_{254}$, Merck grade was used for thin layer chromatography to determine the progress of the reaction. Column chromatography was carried out over silica gel (80-120 mesh). The optical rotation was measured on a Polax-2L digital polarimeter. The melting point was determined in an open capillary tube and was uncorrected. ${ }^{1} \mathrm{H}$ and ${ }^{13} \mathrm{C}$ NMR spectra were recorded on an Avance $300 \mathrm{MHz}$ spectrometer using $\mathrm{CDCl}_{3}$ solvent. Mass spectra were obtained on a Polaris-Q Thermoscientific GC-MS. Elemental analyses were obtained using a flash EA 1112. Enantiomeric purity was determined on a PerkinElmer Series 200 HPLC System.
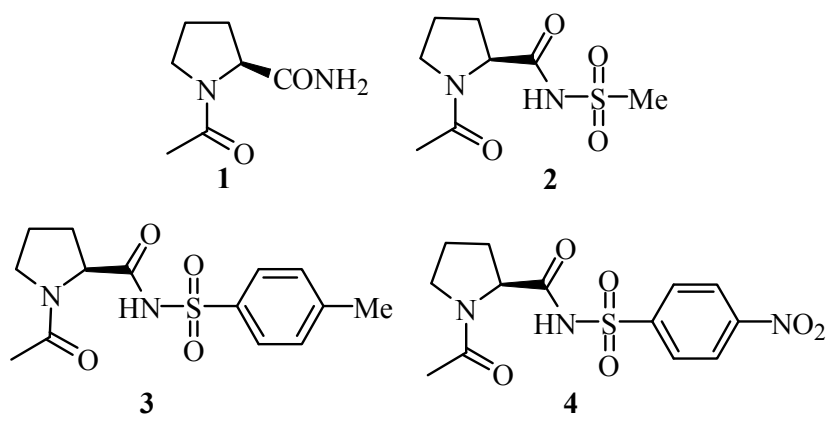

Fig. 1. Pyrrolidine based organocatalysts.

\subsection{Preparation of organocatalysts 1-4}

The synthesis and characterization of the organocatalyst was described in our previous communication [23].

\subsection{General procedure for the aldol reaction for the synthesis of 7a-7f}

To a solution of chloroacetone 6 (1.5 eq.) dissolved in ethanol, a few drops of triethylamine (3-4 drops) was added and the reaction mixture was stirred for 15-20 min. To this stirred solution, an aromatic aldehyde 5(a-f) (1.0 eq.) and a catalytic amount of the organocatalyst $(S)$-1-acetyl- $N$-tosylpyrrolidine2-carboxamide $\mathbf{3}$ was added and reacted and stirred for an appropriate time (Table 2). The progress of reaction was monitored by thin layer chromatography. After completion of the reaction, the solvent was evaporated under vacuum. The crude product was partitioned between ethyl acetate and water. The organic layer was collected, and the aqueous phase was extracted with ethyl acetate. The collected organic layer was washed with saturated brine solution, and the product was purified using column chromatography using silica gel (80-120 mesh).

3-Chloro-4-hydroxy-4-(4-nitrophenyl)butan-2-one (7a). Light yellow solid (182 mg, 90\%), M.P. $142-144{ }^{\circ} \mathrm{C}$ [19]; ${ }^{1} \mathrm{H}$ NMR (300 MHz, $\left.\mathrm{CDCl}_{3}\right): \delta 7.40-7.52(\mathrm{~m}, 2 \mathrm{H}), 7.12-7.28(\mathrm{~m}, 2 \mathrm{H})$, 6.01 and 5.76 (bs, 1H, OH for syn and anti), 5.21 (s, 1H) syn, $4.96(\mathrm{~d}, 1 \mathrm{H}, J=8.7 \mathrm{~Hz})$ anti, $4.29(\mathrm{~s}, 1 \mathrm{H})$ anti, $3.95(\mathrm{~s}, 1 \mathrm{H})$ syn, 2.08 (s, 3H); ${ }^{13} \mathrm{C}$ NMR (300 MHz, $\mathrm{CDCl}_{3}$ ): $\delta 209.9,154.5,149.8$, 129.7, 123.2, 68.9, 59.1, 28.1; GC-MS m/z 243 (M+); Elemental analysis: Anal. Calcd for $\mathrm{C}_{10} \mathrm{H}_{10} \mathrm{ClNO}_{4}$ : C, 49.30; $\mathrm{H}, 4.14 ; \mathrm{Cl}$, 14.55; N, 5.75; 0, 26.27; Found C, 49.31; H, 4.17; Cl, 14.57; N, 5.76; 0, 26.29. Enantiomeric purity was determined by chiral HPLC using Whelk-01 (25 cm $\times 4.6 \mathrm{~mm}), 20: 80: 0.5 \mathrm{IPA} / \mathrm{hex}-$ ane/HOAc, flow rate $1.0 \mathrm{~mL} / \mathrm{min}, \lambda=254 \mathrm{~nm}$; $t_{\mathrm{R}}$ (major) $=17.2$ $\min , t_{\mathrm{R}}$ (minor) $=25.5 \mathrm{~min}, e e=92 \%$.

3-Chloro-4-(4-fluorophenyl)-4-hydroxybutan-2-one (7b). Colorless solid, (158 mg, 92\%), M.P. $77-79{ }^{\circ} \mathrm{C}$; ${ }^{1} \mathrm{H}$ NMR (300 $\left.\mathrm{MHz}_{\mathrm{CDCl}}\right): \delta 7.56-7.66(\mathrm{~m}, 2 \mathrm{H}), 7.23-7.34(\mathrm{~m}, 2 \mathrm{H}), 5.79$ and 5.46 (bs, $1 \mathrm{H}, \mathrm{OH}$ for syn and anti), $5.21(\mathrm{~s}, 1 \mathrm{H})$ syn, $4.88(\mathrm{~d}, 1 \mathrm{H}, J$ $=8.2 \mathrm{~Hz})$ anti, $4.33(\mathrm{~s}, 1 \mathrm{H})$ anti, $3.89(\mathrm{~s}, 1 \mathrm{H})$ syn, $1.98(\mathrm{~s}, 3 \mathrm{H}) ;{ }^{13} \mathrm{C}$ NMR (300 MHz, $\left.\mathrm{CDCl}_{3}\right): \delta 208.4,151.8,147.6,128.9,124.5$, 70.4, 57.9, 29.1; GC-MS m/z 246 (M+); Elemental analysis: Anal. Calcd for $\mathrm{C}_{11} \mathrm{H}_{12} \mathrm{ClFO}_{3}$ : C, 53.56; $\mathrm{H}, 4.90 ; \mathrm{Cl}, 14.37 ; \mathrm{F}, 7.70 ; \mathrm{O}$, 19.46; Found C, 53.54; H, 4.91; Cl, 14.40; F, 7.73; 0, 19.44. Enantiomeric purity was determined by chiral HPLC using Whelk-01 (25 cm $\times 4.6 \mathrm{~mm}$ ), 20:80:0.5 IPA/hexane/HOAc, flow rate $1.0 \mathrm{~mL} / \mathrm{min}, \lambda=254 \mathrm{~nm}$, $t_{\mathrm{R}}$ (major) $=23.2 \mathrm{~min}, t_{\mathrm{R}}($ minor $)=$ $27.1 \mathrm{~min}, e e=92 \%$.

4-(2-Chloro-1-hydroxy-3-oxobutyl)benzonitrile (7c). Viscous oil (171 mg, 88\%) [19]; ${ }^{1} \mathrm{H}$ NMR (300 $\mathrm{MHz}^{\mathrm{CDCl}} 3$ ): $\delta$ 7.56-7.94 (m, 4H), 6.14 and 5.82 (bs, $1 \mathrm{H}, \mathrm{OH}$ for syn and anti), $5.61(\mathrm{~s}, 1 \mathrm{H})$ syn, $4.37(\mathrm{~d}, 1 \mathrm{H}, J=9.3 \mathrm{~Hz})$ anti, $4.45(\mathrm{~s}, 1 \mathrm{H})$ anti, 3.95 (s, 1H) syn, 2.17 (s, 3H); ${ }^{13} \mathrm{C}$ NMR (300 MHz, $\left.\mathrm{CDCl}_{3}\right): \delta$ 205.1, 149.1, 132.7, 128.3, 112.2, 106.6, 81.1, 64.4, 23.4; GC-MS $m / z 223(\mathrm{M}+)$; Elemental analysis: Anal. Calcd for $\mathrm{C}_{11} \mathrm{H}_{10} \mathrm{ClNO}_{2}$ : C, 59.07; H, 4.51; Cl, 15.85; N, 6.26; 0, 14.31; Found C, 59.10; H, 
4.55; Cl, 15.87; N, 6.29; 0, 14.33. Enantiomeric purity was determined by chiral HPLC using Whelk-01 $(25 \mathrm{~cm} \times 4.6 \mathrm{~mm})$, 20:80:0.5 IPA/hexane/HOAc, flow rate $1.0 \mathrm{~mL} / \mathrm{min}, \lambda=254$ $\mathrm{nm} ; t_{\mathrm{R}}$ (major) $=24.2 \mathrm{~min}, t_{\mathrm{R}}$ (minor) $=31.4 \mathrm{~min}, e e=90 \%$.

3-Chloro-4-(4-chlorophenyl)-4-hydroxybutan-2-one (7d). White sticky solid (165 mg, 85\%); ${ }^{1} \mathrm{H}$ NMR (300 MHz, $\mathrm{CDCl}_{3}$ ): $\delta$ 7.26-7.36 (m, 4H), 6.03 and 5.79 (bs, $1 \mathrm{H}, \mathrm{OH}$ for syn and anti), $5.34(\mathrm{~s}, 1 \mathrm{H})$ syn, $4.41(\mathrm{~d}, 1 \mathrm{H}, J=8.4 \mathrm{~Hz})$ anti, $4.36(\mathrm{~s}, 1 \mathrm{H})$ anti, 4.03 (s, 1H) syn, $2.14(\mathrm{~s}, 3 \mathrm{H}) ;{ }^{13} \mathrm{C}$ NMR (300 MHz, $\left.\mathrm{CDCl}_{3}\right): \delta$ 203.4, 153.3, 147.1, 129.2, 126.7, 71.0, 59.8, 29.5; GC-MS $m / z$ $263(\mathrm{M}+)$; Elemental analysis: Anal. Calcd for $\mathrm{C}_{11} \mathrm{H}_{12} \mathrm{Cl}_{2} \mathrm{O}_{3}$ : C, 50.21; H, 4.60; Cl, 26.95; 0, 18.24; Found C, 50.24; H, 4.63; Cl, 26.91; 0, 18.22. Enantiomeric purity was determined by chiral HPLC using Whelk-01 (25 cm $\times 4.6 \mathrm{~mm}), 20: 80: 0.5$ IPA/hexane/HOAc, flow rate $1.0 \mathrm{~mL} / \mathrm{min}, \lambda=254 \mathrm{~nm}$; $t_{\mathrm{R}}$ (major) $=20.2$ $\min , t_{\mathrm{R}}$ (minor) $=27.3 \mathrm{~min}, e e=88 \%$.

4-(2-Bromophenyl)-3-chloro-4-hydroxybutan-2-one (7e). Brownish oil (178 mg, 77\%) [24]; ${ }^{1} \mathrm{H}$ NMR (300 MHz, $\left.\mathrm{CDCl}_{3}\right): \delta$ 7.26-7.35 (m, 2H), 7.19-7.25 (m, 1H), 7.05-7.12 (m, 1H), 5.74 and 5.51 (bs, 1H, OH for syn and anti), $5.19(\mathrm{~s}, 1 \mathrm{H})$ syn, $4.93(\mathrm{~d}$, $1 \mathrm{H}, J=7.2 \mathrm{~Hz}$ ) anti, $4.40(\mathrm{~s}, 1 \mathrm{H})$ anti, $3.85(\mathrm{~s}, 1 \mathrm{H})$ syn, $2.11(\mathrm{~s}$, $3 \mathrm{H}) ;{ }^{13} \mathrm{C}$ NMR (300 MHz, $\mathrm{CDCl}_{3}$ ): $\delta 210.3,153.6,148.3,127.2$, 126.5, 71.8, 59.4, 30.4; GC-MS m/z 307 (M+); Elemental analysis: Anal. Calcd for $\mathrm{C}_{11} \mathrm{H}_{12} \mathrm{BrClO}_{3}$ : C, 42.96; $\mathrm{H}, 3.93 ; \mathrm{Br}, 25.98 ; \mathrm{Cl}$, 11.53; 0, 15.61; Found C, 42.96; H, 3.93; Br, 25.98; Cl, 11.53; O, 15.61. Enantiomeric purity was determined by chiral HPLC using Whelk-01 (25 cm $\times 4.6 \mathrm{~mm}), 20: 80: 0.5 \mathrm{IPA} / \mathrm{hexane} /$ HOAc, flow rate $1.0 \mathrm{~mL} / \mathrm{min}, \lambda=254 \mathrm{~nm} ; t_{\mathrm{R}}$ (major) $=23.4 \mathrm{~min}$, $t_{\mathrm{R}}($ minor $)=25.1 \mathrm{~min}, e e=84 \%$.

3-Chloro-4-hydroxy-4-phenylbutan-2-one (7f). Colorless solid (120 mg, 73\%), M.P. $110-112{ }^{\circ} \mathrm{C}$ [24]; ${ }^{1} \mathrm{H}$ NMR $(300 \mathrm{MHz}$, $\left.\mathrm{CDCl}_{3}\right): \delta 6.97-7.43(\mathrm{~m}, 4 \mathrm{H}), 5.84$ and 5.51 (bs, $1 \mathrm{H}, \mathrm{OH}$ for syn and anti), $5.17(\mathrm{~s}, 1 \mathrm{H})$ syn, $4.92(\mathrm{~d}, 1 \mathrm{H}, 7.5 \mathrm{~Hz})$ anti, $4.27(\mathrm{~s}, 1 \mathrm{H})$ anti, 4.01 (s, 1H) syn, $\left.2.04(\mathrm{~s}, 3 \mathrm{H}) ;{ }^{13} \mathrm{C} \mathrm{NMR} \mathrm{(300} \mathrm{MHz,} \mathrm{CDCl}_{3}\right): \delta$ 203.4, 153.3, 145.5, 123.2, 123.1, 71.4, 60.0, 31.1; GC-MS $m / z$ $228(\mathrm{M}+)$; Elemental analysis: Anal. Calcd for $\mathrm{C}_{11} \mathrm{H}_{13} \mathrm{ClO}_{3}: \mathrm{C}$, 57.78; H, 5.73; Cl, 15.50; O, 20.99; Found C, 57.80; H, 5.74; Cl, 15.52; 0, 20.96. Enantiomeric purity was determined by chiral HPLC using Whelk-01 (25 cm $\times 4.6 \mathrm{~mm}), 20: 80: 0.5$ IPA/hexane $/ \mathrm{HOAc}$, flow rate $1.0 \mathrm{~mL} / \mathrm{min}, \lambda=254 \mathrm{~nm} ; t_{\mathrm{R}}$ (major) $=27.4$ $\min , t_{\mathrm{R}}($ minor $)=28.2 \mathrm{~min}, e e=86 \%$.

\subsection{General procedure for the Knoevenagel condensation reaction (9a-9j)}

To a solution of ethylcyanoacetate 8 (1.5 eq.) dissolved in ethanol, a few drops of triethylamine (3-4 drops) was added and the reaction mixture was stirred for 15-20 min. To this stirred solution, an aromatic aldehyde 5(a-f) (1.0 eq.) and a catalytic amount of the organocatalyst $(S)$-1-acetyl- $N$-tosylpyrrolidine-2-carboxamide $\mathbf{3}$ in ethanol was added. The reaction mixture was stirred at $50{ }^{\circ} \mathrm{C}$ for an appropriate time (Table 4). The progress of reaction was monitored by thin layer chromatography. After completion of the reaction, the mixture was cooled and the solvent was evaporated under vacuum. The crude product was partitioned between ethyl acetate and water. The organic layer was collected and the aqueous phase was extracted with ethyl acetate. The collected organic layer was washed with saturated brine solution and the product was purified using column chromatography using silica gel (80-120 mesh).

Ethyl 2-cyano-3-(4-nitrophenyl)acrylate (9a). Light yellow solid (261 mg, 79\%), M.P. $168-170{ }^{\circ} \mathrm{C}$ [25]; ${ }^{1} \mathrm{H}$ NMR $(300 \mathrm{MHz}$, $\left.\mathrm{CDCl}_{3}\right): \delta 8.27(\mathrm{~s}, 1 \mathrm{H}), 7.12-7.28(\mathrm{~m}, 2 \mathrm{H}), 7.69-7.79(\mathrm{~m}, 2 \mathrm{H})$, 3.98 (q, 2H), 1.38 (t, 3H); ${ }^{13} \mathrm{C}$ NMR (300 MHz, $\left.\mathrm{CDCl}_{3}\right): \delta 165.03$, 151.00, 146.65, 129.71, 122.58, 119.74, 117.18, 101.69, 59.21, 18.56; GC-MS m/z 246 (M+); Elemental analysis: Anal. Calcd for $\mathrm{C}_{12} \mathrm{H}_{10} \mathrm{~N}_{2} \mathrm{O}_{4}$ : C, 58.54; H, 4.09; N, 11.38; 0, 25.99; Found C, 58.53; H, 4.10; N, 11.36; 0, 25.97.

Ethyl 2-cyano-3-(3-nitrophenyl)acrylate (9b). Yellow solid (240 mg, 78\%), M.P. $133-135{ }^{\circ} \mathrm{C}[26] ;{ }^{1} \mathrm{H}$ NMR $(300 \mathrm{MHz}$, $\left.\mathrm{CDCl}_{3}\right): \delta 8.19(\mathrm{~s}, 1 \mathrm{H}), 8.21-8.26(\mathrm{~m}, 1 \mathrm{H}), 7.91-7.98(\mathrm{~m}, 1 \mathrm{H})$, 7.81-7.89 (m, 2H), $4.12(\mathrm{q}, 2 \mathrm{H}), 1.25(\mathrm{t}, 3 \mathrm{H}) ;{ }^{13} \mathrm{C}$ NMR $(300$ $\left.\mathrm{MHz} \mathrm{CDCl}_{3}\right): \delta 162.6,152.30,147.33,132.91,128.25,126.81$, 126.11, 122.61, 117.18, 105.16, 61.42, 14.87; GC-MS m/z 246 $(\mathrm{M}+)$; Elemental analysis: Anal. Calcd for $\mathrm{C}_{12} \mathrm{H}_{10} \mathrm{~N}_{2} \mathrm{O}_{4}$ : C, 58.54; H, 4.09; N, 11.38; 0, 25.99; Found C, 58.51; H, 4.11; N, 11.35; 0, 26.02 .

Ethyl 2-cyano-3-(4-fluorophenyl)acrylate (9c) (CAS No. 18861-57-9). Yellow crystalline (135 mg, 79\%), M.P. $96-98{ }^{\circ} \mathrm{C}$; ${ }^{1} \mathrm{H}$ NMR (300 MHz, CDCl 3 ): $\delta 8.42(\mathrm{~s}, 1 \mathrm{H}), 7.01-7.19(\mathrm{~m}, 2 \mathrm{H})$, 7.82-8.10 (m, 2H), $4.26(\mathrm{q}, 2 \mathrm{H}), 1.60(\mathrm{t}, 3 \mathrm{H}) ;{ }^{13} \mathrm{C}$ NMR (300 $\left.\mathrm{MHz}, \mathrm{CDCl}_{3}\right): \delta 163.51,162.24,153.33,126.03,119.68,117.27$, 98.64, 55.87, 22.31; GC-MS m/z 219 (M+); Elemental analysis: Anal. Calcd for $\mathrm{C}_{12} \mathrm{H}_{10} \mathrm{FNO}_{2}$ : C, 65.75; H, 4.60; F, 8.67; N, 6.39; O, 14.60; Found C, 65.71; H, 4.63; F, 8.68; N, 6.40; 0, 14.61.

Ethyl 2-cyano-3-(4-cyanophenyl)acrylate (9d). Colorless solid (244 mg, 80\%), M.P. $133-135{ }^{\circ} \mathrm{C}$; ${ }^{1} \mathrm{H}$ NMR $(300 \mathrm{MHz}$, $\left.\mathrm{CDCl}_{3}\right): \delta 8.34(\mathrm{~s}, 1 \mathrm{H}), 7.67-7.73(\mathrm{~m}, 2 \mathrm{H}), 7.42-8.61(\mathrm{~m}, 2 \mathrm{H})$, $4.23(\mathrm{q}, 2 \mathrm{H}), 1.34(\mathrm{t}, 3 \mathrm{H}) ;{ }^{13} \mathrm{C}$ NMR (300 MHz, $\left.\mathrm{CDCl}_{3}\right): \delta 161.54$, 157.43, 138.61, 130.91, 129.17, 117.34, 117.02, 109.76, 99.97, 63.12, 15.43; GC-MS m/z $226(\mathrm{M}+)$; Elemental analysis: Anal. Calcd for $\mathrm{C}_{13} \mathrm{H}_{10} \mathrm{~N}_{2} \mathrm{O}_{2}$ : C, 69.02; $\mathrm{H}, 4.46 ; \mathrm{N}, 12.38 ; 0,14.14$; Found C, 68.99; H, 4.49; N, 12.36; 0, 14.12 .

Ethyl 3-(4-chlorophenyl)-2-cyanoacrylate (9e). Brownish solid (240 mg, 76\%), M.P. 92-94 ${ }^{\circ} \mathrm{C}$ [27]; ${ }^{1} \mathrm{H}$ NMR $(300 \mathrm{MHz}$, $\left.\mathrm{CDCl}_{3}\right): \delta 8.19(\mathrm{~s}, 1 \mathrm{H}), 7.82-7.88(\mathrm{~m}, 2 \mathrm{H}), 7.12-8.23(\mathrm{~m}, 2 \mathrm{H})$, $4.18(\mathrm{q}, 2 \mathrm{H}), 1.28(\mathrm{t}, 3 \mathrm{H}) ;{ }^{13} \mathrm{C}$ NMR $\left(300 \mathrm{MHz}, \mathrm{CDCl}_{3}\right): \delta 162.71$, 152.13, 135.37, 134.89, 131.29, 130.27, 118.35, 101.49, 62.94, 14.99; GC-MS m/z 235 (M+); Elemental analysis: Anal. Calcd for $\mathrm{C}_{12} \mathrm{H}_{10} \mathrm{ClNO}_{2}$ : C, 61.16; $\mathrm{H}, 4.28 ; \mathrm{Cl}, 15.04 ; \mathrm{N}, 5.94 ; 0,13.58$; Found C, 61.14; H, 4.31; Cl, 15.02; N, 5.96; 0, 13.61.

Ethyl 3-(2-chlorophenyl)-2-cyanoacrylate (9f). Reddish solid (231 mg, 73\%), M.P. 54-55 ${ }^{\circ} \mathrm{C}$ [27]; ${ }^{1} \mathrm{H}$ NMR $(300 \mathrm{MHz}$, $\left.\mathrm{CDCl}_{3}\right): \delta 8.11(\mathrm{~s}, 1 \mathrm{H}), 7.54-7.49(\mathrm{~m}, 1 \mathrm{H}), 7.31-8.44(\mathrm{~m}, 2 \mathrm{H})$, $4.23(\mathrm{q}, 2 \mathrm{H}), 1.32(\mathrm{t}, 3 \mathrm{H}) ;{ }^{13} \mathrm{C}$ NMR $\left(300 \mathrm{MHz}, \mathrm{CDCl}_{3}\right): \delta 162.71$, 150.84, 134.76, 130.41, 129.65, 129.01, 128.77, 116.97, 104.42, 63.24, 15.43; GC-MS m/z $235(\mathrm{M}+)$; Elemental analysis: Anal. Calcd for $\mathrm{C}_{12} \mathrm{H}_{10} \mathrm{ClNO}_{2}$ : C, 61.16; $\mathrm{H}, 4.28 ; \mathrm{Cl}, 15.04 ; \mathrm{N}, 5.94 ; \mathrm{O}$, 13.58; Found C, 61.13; H, 4.29; Cl, 15.03; N, 5.93; 0, 13.60.

Ethyl 2-cyano-3-phenylacrylate (9g). White solid $(210 \mathrm{mg}$, 72\%), M.P. $51-53{ }^{\circ} \mathrm{C}$ [28]; ${ }^{1} \mathrm{H}$ NMR (300 MHz, $\left.\mathrm{CDCl}_{3}\right): \delta 8.24(\mathrm{~s}$, 1H), 7.33-7.39 (m, 1H), 7.33-8.10 (m, 4H), $4.20(\mathrm{q}, 2 \mathrm{H}), 1.27(\mathrm{t}$, $3 \mathrm{H})$; ${ }^{13} \mathrm{C}$ NMR (300 MHz, $\left.\mathrm{CDCl}_{3}\right): \delta 160.21,154.11,133.54$, 
132.87, 129.75, 129.22, 128.77, 117.16, 101.59, 60.89, 14.87; GC-MS $m / z 201(\mathrm{M}+)$; Elemental analysis: Anal. Calcd for $\mathrm{C}_{12} \mathrm{H}_{11} \mathrm{NO}_{2}$ : C, 71.63; H, 5.51; N, 6.96; 0, 15.90; Found C, 71.63; H, 5.51; N, 6.96; 0, 15.90 .

Ethyl 2-cyano-3-p-tolylacrylate (9h). White solid (203 mg, 74\%), M.P. 90-92 ${ }^{\circ} \mathrm{C}$ [29]; ${ }^{1} \mathrm{H}$ NMR (300 MHz, $\mathrm{CDCl}_{3}$ ): $\delta 8.17$ (s, $1 \mathrm{H}), 7.76-8.88(\mathrm{~m}, 2 \mathrm{H}), 7.28-7.39(\mathrm{~m}, 2 \mathrm{H}), 4.01(\mathrm{q}, 2 \mathrm{H}), 2.40(\mathrm{~s}$, $3 \mathrm{H}), 1.31(\mathrm{t}, 3 \mathrm{H}) ;{ }^{13} \mathrm{C} \mathrm{NMR}\left(300 \mathrm{MHz}, \mathrm{CDCl}_{3}\right): \delta 161.91,151.77$, 142.81, 122.72, 122.68, 119.27, 99.99, 60.08, 23.19, 16.29; GC-MS $m / z 215(\mathrm{M}+)$; Elemental analysis: Anal. Calcd for $\mathrm{C}_{13} \mathrm{H}_{13} \mathrm{NO}_{2}$ : C, 72.54; H, 6.09; N, 6.51; 0, 14.87; Found C, 72.56; H, 6.06; N, 6.53; 0, 14.85 .

Ethyl 2-cyano-3-(4-hydroxyphenyl)acrylate (9i). Reddish solid (203 mg, 69\%), M.P. $172-174{ }^{\circ} \mathrm{C}[30]$; ${ }^{1} \mathrm{H}$ NMR $(300 \mathrm{MHz}$, $\mathrm{CDCl}_{3}$ ): $\delta 9.28$ (bs, $\left.1 \mathrm{H}, \mathrm{OH}\right), 8.68(\mathrm{~s}, 1 \mathrm{H}), 8.21-8.40(\mathrm{~m}, 2 \mathrm{H})$, 7.28-7.71 (m, 2H), 4.08 (q, 2H), $1.26(\mathrm{t}, 3 \mathrm{H})$; ${ }^{13} \mathrm{C}$ NMR $(300$ $\left.\mathrm{MHz}, \mathrm{CDCl}_{3}\right): \delta 163.47,159.83,150.01,138.30,127.61,121.89$, 117.26, 108.05, 61.87, 23.24; GC-MS m/z 217 (M+); Elemental analysis: Anal. Calcd for $\mathrm{C}_{12} \mathrm{H}_{11} \mathrm{NO}_{3}: \mathrm{C}, 66.35 ; \mathrm{H}, 5.10 ; \mathrm{N}, 6.45 ; \mathrm{O}$, 22.10; Found C, 66.33; H, 5.12; N, 6.43; 0, 22.11.

Ethyl 2-cyano-3-(3,4-dimethoxyphenyl)acrylate (9j). Brownish solid (234 mg, 67\%), M.P. $56-58{ }^{\circ} \mathrm{C}$ [31]; ${ }^{1} \mathrm{H}$ NMR (300 MHz, CDCl $): \delta 8.23$ (s, 1H), 7.23-7.10 (m, 2H), 7.01-7.06 $(\mathrm{m}, 1 \mathrm{H}), 4.16(\mathrm{q}, 2 \mathrm{H}), 3.90(\mathrm{~s}, 6 \mathrm{H}), 1.31(\mathrm{t}, 3 \mathrm{H}) ;{ }^{13} \mathrm{C}$ NMR $(300$ $\mathrm{MHz}_{\mathrm{CDCl}}$ ): $\delta 164.09,151.85,150.31,149.78,129.65,123.76$, 117.08, 116.98, 116.31, 102.37, 60.45, 58.93, 17.62; GC-MS $m / z$ $261(\mathrm{M}+)$; Elemental analysis: Anal. Calcd for $\mathrm{C}_{14} \mathrm{H}_{15} \mathrm{NO}_{4}: \mathrm{C}$, 64.36; H, 5.79; N, 5.36; 0, 24.49; Found C, 64.34; H, 5.81; N, $5.39 ; 0,24.47$.

\subsection{General procedure for the synthesis of hydroxy propanoate derivatives (10a-10j)}

To a solution of ethylcyanoacetate 8 (1.5 eq.) dissolved in ethanol, a few drops of triethylamine (3-4 drops) was added and the reaction mixture was stirred for 15-20 min. To this stirred cold solution, an aromatic aldehyde 5(a-f) (1.0 equivalent) and a catalytic amount of the organocatalyst $(S)$-1-acetyl$\mathrm{N}$-tosylpyrrolidine-2-carboxamide $\mathbf{3}$ in ethanol was added. The reaction mixture was cooled to $-78{ }^{\circ} \mathrm{C}$ and stirred for an appropriate time (Table 4). The progress of reaction was monitored by thin layer chromatography. After completion of the reaction, crushed ice was added to this cold solution and the mixture was allowed to stand. The reaction mixture was extracted with ethyl acetate. The collected organic layer was washed with saturated brine solution and the product was purified using column chromatography using silica gel (80-120 mesh).

Ethyl 2-cyano-3-(4-nitrophenyl)-3-hydroxypropanoate (10a). Yellow viscous oil (274 mg, 89\%); ${ }^{1} \mathrm{H}$ NMR (300 MHz, $\left.\mathrm{CDCl}_{3}\right): \delta$ 7.48-60 (m, 2H), 7.19-7.30 (m, 2H), 5.17 (bs, 1H, OH), 4.44 (d, $1 \mathrm{H}), 4.31(\mathrm{q}, 2 \mathrm{H}), 3.87(\mathrm{~d}, 1 \mathrm{H}), 1.32(\mathrm{t}, 3 \mathrm{H}) ;{ }^{13} \mathrm{C}$ NMR $(300 \mathrm{MHz}$, $\left.\mathrm{CDCl}_{3}\right): \delta 163.85,149.97,148.69,130.78,125.63,117.11,74.83$, 58.23, 39.00, 16.91; GC-MS m/z $264(\mathrm{M}+)$; HRMS: Calculated 264.0746; Found 264.0744; Elemental analysis: Anal. Calcd for $\mathrm{C}_{12} \mathrm{H}_{12} \mathrm{~N}_{2} \mathrm{O}_{5}$ : C, 54.55; H, 4.58; N, 10.60; O, 30.28; Found $\mathrm{C}$, 54.55; H, 4.58; N, 10.60; 0, 30.28. Enantiomeric purity was de- termined by chiral HPLC using Whelk-01 $(25 \mathrm{~cm} \times 4.6 \mathrm{~mm})$, 20:80:0.5 IPA/hexane/HOAc, flow rate $1.0 \mathrm{~mL} / \mathrm{min}, \lambda=254$ $\mathrm{nm} ; t_{\mathrm{R}}$ (major) $=7.7 \mathrm{~min}, t_{\mathrm{R}}($ minor $)=15.2 \mathrm{~min}, e e=81 \%$.

Ethyl 2-cyano-3-(3-nitrophenyl)-3-hydroxypropanoate (10b). Yellow viscous oil (261 mg, 85\%); ${ }^{1} \mathrm{H}$ NMR (300 $\mathrm{MHz}^{\mathrm{CDCl}} 3$ ): $\delta$ 7.27-7.35 (m, 2H), 7.21-7.26 (m, 1H), 7.05-7.17 (m, 1H), 5.19 (bs, 1H, OH), 4.39 (d, 1H), $4.37(\mathrm{q}, 2 \mathrm{H}), 3.91(\mathrm{~d}, 1 \mathrm{H}), 1.37$ (t, 3H); ${ }^{13} \mathrm{C}$ NMR (300 MHz, $\mathrm{CDCl}_{3}$ ): $\delta 164.33,150.02,143.32,131.78$, 130.65, 125.63, 123.81, 113.01, 72.64, 59.44, 41.21, 18.92; GC-MS $\mathrm{m} / \mathrm{z} 264 \mathrm{M}+$ ); HRMS: Calculated 264.0746; Found 264.0747; Elemental analysis: Anal. Calcd for $\mathrm{C}_{12} \mathrm{H}_{12} \mathrm{~N}_{2} \mathrm{O}_{5}$ : C, 54.55; H, 4.58; N, 10.60; O, 30.28; Found C, 54.55; H, 4.58; N, 10.60; 0, 30.28. Enantiomeric purity was determined by chiral HPLC using Whelk-01 (25 cm $\times 4.6 \mathrm{~mm}), 20: 80: 0.5 \mathrm{IPA} / \mathrm{hex}-$ ane/HOAc, flow rate $1.0 \mathrm{~mL} / \mathrm{min}, \lambda=254 \mathrm{~nm}$; $t_{\mathrm{R}}$ (major) $=9.0$ $\min , t_{\mathrm{R}}$ (minor) $=15.7 \mathrm{~min}, e e=84 \%$.

Ethyl 2-cyano-3-hydroxy-3-(4-flurophenyl) propanoate (10c). Colorless oil (238 mg, 87\%); ${ }^{1} \mathrm{H}$ NMR (300 $\left.\mathrm{MHz} \mathrm{CDCl}_{3}\right): \delta$ 7.94-8.29 (m, 2H), 7.18-7.29 (m, 2H), 5.28 (bs, 1H, OH), 4.90 (d, 1H), 4.30 (q, 2H), $3.31(\mathrm{~d}, 1 \mathrm{H}), 1.29$ (t, 3H); ${ }^{33} \mathrm{C}$ NMR (300 $\mathrm{MHz}, \mathrm{CDCl}_{3}$ ): $\delta 166.19,165.51,130.11,127.03,126.02,110.13$, 71.58, 66.70, 46.42, 14.59; GC-MS m/z 237 (M+); HRMS: Calculated 237.0801; Found 237.0802; Elemental analysis: Anal. Calcd for $\mathrm{C}_{12} \mathrm{H}_{12} \mathrm{FNO}_{3}$ : C, 60.76; H, 5.10; F, 8.01; N, 5.90; O, 20.23; Found C, 60.74; H, 5.13; F, 7.99; N, 5.93; 0, 20.26. Enantiomeric purity was determined by chiral HPLC using Whelk-01 (25 cm $\times 4.6 \mathrm{~mm}$ ), 20:80:0.5 IPA/hexane/HOAc, flow rate $1.0 \mathrm{~mL} / \mathrm{min}, \lambda=254 \mathrm{~nm}$, $t_{\mathrm{R}}$ (major) $=13.3 \mathrm{~min}, t_{\mathrm{R}}$ (minor) $=$ $15.3 \mathrm{~min}, e e=82 \%$.

Ethyl 2-cyano-3-(4-cyanophenyl)-3-hydroxypropanoate (10d). Colorless oil (249 mg, 89\%); ${ }^{1} \mathrm{H}$ NMR (300 $\mathrm{MHz}^{\mathrm{CDCl}} 3$ ): $\delta$ 7.32-7.57 (m, 4H), 5.32 (bs, 1H, OH), 4.87 (d, 1H), 4.35 (q, 2H), $3.31(\mathrm{~d}, 1 \mathrm{H}), 1.31(\mathrm{t}, 3 \mathrm{H}) ;{ }^{13} \mathrm{C} \mathrm{NMR}\left(300 \mathrm{MHz}, \mathrm{CDCl}_{3}\right): \delta 165.66$, 147.39, 133.87, 127.03, 124.38, 120.57, 112.59, 71.56, 68.93, 42.67, 16.74; GC-MS $m / z \quad 244 \quad(\mathrm{M}+)$; HRMS: Calculated 244.0848; Found 244.0850; Elemental analysis: Anal. Calcd for $\mathrm{C}_{13} \mathrm{H}_{12} \mathrm{~N}_{2} \mathrm{O}_{3}$ : C, 63.93; $\mathrm{H}, 4.95 ; \mathrm{N}, 11.47 ; 0,19.65$; Found $\mathrm{C}$, 63.91; H, 4.98; N, 11.44; 0, 19.63. Enantiomeric purity was determined by chiral HPLC using Whelk-01 $(25 \mathrm{~cm} \times 4.6 \mathrm{~mm})$, 20:80:0.5 IPA/hexane/HOAc, flow rate $1.0 \mathrm{~mL} / \mathrm{min}, \lambda=254$ $\mathrm{nm} ; t_{\mathrm{R}}$ (major) $=19.1 \mathrm{~min}, t_{\mathrm{R}}($ minor $)=19.9 \mathrm{~min}, e e=79 \%$.

Ethyl 3-(4-chlorophenyl)-2-cyano-3-hydroxypropanoate (10e). Brown semisolid (247 mg, 84\%); ${ }^{1} \mathrm{H}$ NMR (300 $\mathrm{MHz} \mathrm{CDCl}_{3}$ ): $\delta$ 7.16-7.24 (m, 2H), 7.02-7.11 (m, 2H), 5.35 (bs, 1H, OH), 4.89 (d, 1H), 4.27 (q, 2H), 3.29 (d, 1H), $1.31(\mathrm{t}, 3 \mathrm{H}) ;{ }^{13} \mathrm{C}$ NMR (300 $\mathrm{MHz}, \mathrm{CDCl}_{3}$ ): $\delta 164.22,145.51,137.23,126.02,124.32,117.71$, 69.55, 59.49, 39.41, 16.94; GC-MS m/z 253 (M+); HRMS: Calculated 253.0506; Found 253.0508; Elemental analysis: Anal. Calcd for $\mathrm{C}_{12} \mathrm{H}_{12} \mathrm{ClNO}_{3}$ : C, 56.81; $\mathrm{H}, 4.77 ; \mathrm{Cl}, 13.98 ; \mathrm{N}, 5.52 ; \mathrm{O}$, 18.92; Found C, 56.83; H, 4.78; Cl, 13.95; N, 5.55; 0, 18.93. Enantiomeric purity was determined by chiral HPLC using Whelk-01 (25 cm $\times 4.6 \mathrm{~mm}), 20: 80: 0.5$ IPA/hexane/HOAc, flow rate $1.0 \mathrm{~mL} / \mathrm{min}, \lambda=254 \mathrm{~nm} ; t_{\mathrm{R}}($ major $)=11.6 \mathrm{~min}, t_{\mathrm{R}}($ minor $)=$ $16.4 \mathrm{~min}, e e=83 \%$.

Ethyl 3-(2-chlorophenyl)-2-cyano-3-hydroxypropanoate (10f). Reddish gum (238 mg, 81\%); ${ }^{1} \mathrm{H}$ NMR (300 $\mathrm{MHz}^{\mathrm{CDCl}} 3$ ): $\delta$ 7.51-7.58 (m, 1H), 7.22-7.46 (m, 3H), 5.29 (bs, 1H, OH), 4.89 
(d, 1H), 4.31 (q, 2H), $3.35(\mathrm{~d}, 1 \mathrm{H}), 1.29(\mathrm{t}, 3 \mathrm{H}) ;{ }^{13} \mathrm{C}$ NMR (300 $\mathrm{MHz}_{\mathrm{CDCl}}$ ): $\delta 164.62,144.45,133.28,127.34,127.27,122.63$, 118.84, 63.67, 60.34, 40.27, 15.92; GC-MS $m / z 237$ (M+); HRMS: Calculated 253.0506; Found 253.0507; Elemental analysis: Anal. Calcd for $\mathrm{C}_{12} \mathrm{H}_{12} \mathrm{ClNO}_{3}: \mathrm{C}, 56.81 ; \mathrm{H}, 4.77 ; \mathrm{Cl}, 13.98 ; \mathrm{N}$, 5.52; 0, 18.92; Found C, 56.79; H, 4.79; Cl, 13.96; N, 5.55; O, 18.93. Enantiomeric purity was determined by chiral HPLC using Whelk-01 (25 cm $\times 4.6 \mathrm{~mm}), 20: 80: 0.5$ IPA/hexane/ HOAc, flow rate $1.0 \mathrm{~mL} / \mathrm{min}, \lambda=254 \mathrm{~nm}$; $t_{\mathrm{R}}$ (major) $=14.0 \mathrm{~min}$, $t_{\mathrm{R}}$ (minor) $=20.3 \mathrm{~min}, e e=77 \%$.

Ethyl 2-cyano-3-hydroxy-3-phenylpropanoate (10g). Colorless oil (214 mg, 79\%); ${ }^{1} \mathrm{H}$ NMR (300 $\left.\mathrm{MHz}, \mathrm{CDCl}_{3}\right): \delta$ 7.04-7.48 (m, 4H), 5.33 (bs, 1H, OH), $4.94(\mathrm{~d}, 1 \mathrm{H}), 4.33(\mathrm{q}, 2 \mathrm{H})$, 3.30 (d, 1H), $1.31(\mathrm{t}, 3 \mathrm{H}) ;{ }^{13} \mathrm{C}$ NMR (300 MHz, $\left.\mathrm{CDCl}_{3}\right): \delta 164.42$, 141.32, 126.24, 125.25, 124.89, 117.83, 61.37, 60.07, 40.33, 15.43; GC-MS $m / z 237$ (M+); HRMS: Calculated 219.0895; Found 219.0893; Elemental analysis: Anal. Calcd for $\mathrm{C}_{12} \mathrm{H}_{13} \mathrm{NO}_{3}$ : C, 65.74; H, 5.98; N, 6.39; 0, 21.89; Found C, 65.71; H, 6.00; N, $6.41 ; 0,21.87$. Enantiomeric purity was determined by chiral HPLC using Whelk-01 $(25 \mathrm{~cm} \times 4.6 \mathrm{~mm}), 20: 80: 0.5$ IPA/hexane/HOAc, flow rate $1.0 \mathrm{~mL} / \mathrm{min}, \lambda=254 \mathrm{~nm}$; $t_{\mathrm{R}}$ (major) $=10.1$ $\min , t_{\mathrm{R}}$ (minor) $=11.0 \mathrm{~min}, e e=78 \%$.

Ethyl 2-cyano-3-hydroxy-3-p-tolylpropanoate (10h). White amorphous (201 mg, 80\%); ${ }^{1} \mathrm{H}$ NMR (300 $\mathrm{MHz}^{\left.-\mathrm{CDCl}_{3}\right): \delta}$ 7.34-8.45 (m, 2H), 7.14-7.26 (m, 2H), 5.30 (bs, 1H, OH), 4.95 (d, 1H), 4.31 (q, 2H), $3.83(\mathrm{~d}, 1 \mathrm{H}), 2.68(\mathrm{~s}, 3 \mathrm{H}), 1.25(\mathrm{t}, 3 \mathrm{H}) ;{ }^{13} \mathrm{C}$ NMR (300 MHz, $\left.\mathrm{CDCl}_{3}\right): \delta 162.74,147.75,147.26,122.09$, 120.16, 117.61, 64.32, 58.78, 40.69, 21.73, 13.58; GC-MS $m / z$ $233(\mathrm{M}+)$; HRMS: Calculated 235.0845; Found 235.0844; Elemental analysis: Anal. Calcd for $\mathrm{C}_{13} \mathrm{H}_{15} \mathrm{NO}_{3}$ : C, 66.94; $\mathrm{H}, 6.48 ; \mathrm{N}$, 6.00; 0, 20.58; Found C, 66.97; H, 6.45; N, 5.97; 0, 20.59. Enantiomeric purity was determined by chiral HPLC using Whelk-01 (25 cm $\times 4.6 \mathrm{~mm}$ ), 20:80:0.5 IPA/hexane/HOAc, flow rate $1.0 \mathrm{~mL} / \mathrm{min}, \lambda=254 \mathrm{~nm}$; $t_{\mathrm{R}}$ (major) $=14.6 \mathrm{~min}, t_{\mathrm{R}}$ (minor) $=$ $17.5 \mathrm{~min}, e e=75 \%$.

Ethyl 2-cyano-3-hydroxy-3-(4-hydroxyphenyl) propanoate (10i). Reddish viscous oil (207 mg, 77\%); ${ }^{1} \mathrm{H}$ NMR (300 MHz, $\mathrm{CDCl}_{3}$ ): $\delta$ 7.94-8.29 (m, 2H), 7.18-7.29 (m, 2H), 5.29 (bs, $1 \mathrm{H}$, $\mathrm{OH}), 4.96(\mathrm{~d}, 1 \mathrm{H}), 4.28(\mathrm{q}, 2 \mathrm{H}), 3.73(\mathrm{~d}, 1 \mathrm{H}), 1.26(\mathrm{t}, 3 \mathrm{H}) ;{ }^{13} \mathrm{C}$ NMR (300 MHz, $\left.\mathrm{CDCl}_{3}\right): \delta 165.03,72.34,157.62,140.78$, $122.09,116.28,115.55,60.12,57.08,36.93,12.96$; GC-MS $m / z$ 235 (M+); HRMS: Calculated 279.1107; Found 279.1106; Elemental analysis: Anal. Calcd for $\mathrm{C}_{12} \mathrm{H}_{13} \mathrm{NO}_{4}$ : C, 61.27; H, 5.57; N, 5.95; 0, 27.21; Found C, 61.28; H, 5.59; N, 5.92; 0, 27.24. Enantiomeric purity was determined by chiral HPLC using Whelk-01 (25 cm $\times 4.6 \mathrm{~mm}), 20: 80: 0.5$ IPA/hexane/HOAc, flow rate $1.0 \mathrm{~mL} / \mathrm{min}, \lambda=254 \mathrm{~nm}$; $t_{\mathrm{R}}$ (major) $=12.3 \mathrm{~min}, t_{\mathrm{R}}$ (minor) $=$ $19.2 \mathrm{~min}, e e=76 \%$.

Ethyl 2-cyano-3-hydroxy-3-(3,4-dimethoxyphenyl) propanoate (10j). Brownish oil (233 mg, 73\%); ${ }^{1} \mathrm{H} \mathrm{NMR}(300 \mathrm{MHz}$, $\left.\mathrm{CDCl}_{3}\right): \delta$ 7.57-8.69 (m, 2H), 7.24-7.33 (m, 2H), $5.20(\mathrm{bs}, 1 \mathrm{H}$, $\mathrm{OH}), 4.77(\mathrm{~d}, 1 \mathrm{H}), 4.31(\mathrm{q}, 2 \mathrm{H}), 3.89(\mathrm{~s}, 6 \mathrm{H}), 3.43(\mathrm{~d}, 1 \mathrm{H}), 1.32(\mathrm{t}$, $3 \mathrm{H})$; ${ }^{13} \mathrm{C}$ NMR $\left(300 \mathrm{MHz}, \mathrm{CDCl}_{3}\right): \delta 163.09,157.51,157.13$, $130.91,118.83,109.20,106.00,105.01,78.25,73.14,62.00$, 49.23, 20.50; GC-MS m/z 279 (M+); Elemental analysis: Anal. Calcd for $\mathrm{C}_{14} \mathrm{H}_{17} \mathrm{NO}_{5}$ : C, 60.21; $\mathrm{H}, 6.14 ; \mathrm{N}, 5.02 ; 0,28.64$; Found C, 60.19; H, 6.17; N, 5.04; 0, 28.66. Enantiomeric purity was determined by chiral HPLC using Whelk-01 (25 cm $\times 4.6 \mathrm{~mm})$, 20:80:0.5 IPA/hexane/HOAc, flow rate $1.0 \mathrm{~mL} / \mathrm{min}, \lambda=254$ $\mathrm{nm} ; t_{\mathrm{R}}$ (major) $=11.0 \mathrm{~min}, t_{\mathrm{R}}($ minor $)=11.4 \mathrm{~min}, e e=76 \%$.

\section{Results and discussion}

In a continuation of our work on organocatalysis [32-36], we explored these chiral organocatalysts in the asymmetric aldol reaction. The aldol reaction of chloroacetone (6) and $p$-nitrobenzaldehyde (5a) to afford $\mathbf{7 a}$ was performed in ethanol using $10 \mathrm{~mol} \%$ of the synthesized organocatalysts 1-4 bearing different sulfonamide groups in the presence of triethylamine (Scheme 1).

Initially, when just $\mathrm{N}$-acylated prolinamide $\mathbf{1}$ was used as the catalyst in the aldol reaction, after stirring for $48 \mathrm{~h}$, we obtained only $29 \%$ yield. The diastereoselectivity and enantioselectivity were not determined for the reaction (Table 1, entry 1). The methanesulfonamide catalyst $\mathbf{2}$ afforded some improved yield (40\%), but the diastereoselectivity and enantiomeric excess were very poor. The best result with respect to yield, diastereoselectivity, and enantioselectivity was with catalyst $\mathbf{3}$ and triethylamine. The reaction with organocatalyst $\mathbf{3}$ gave 7a in good yield with good diastereoselectivity and improved enantioselectivity $69 \%$ ee for anti-7a (Table 1, entry 3). Catalyst 4 catalyzed the aldol reaction affording 59\% yield and decreased diastereoselectivity and enantiomeric excess (Table 1, entries 2-4). Although organocatalysts 3 and $\mathbf{4}$ only showed moderate stereoselectivity, the reaction time was short, esp. with organocatalyst 3 , which completed the reaction within 20 h. Interestingly, the reaction gave enhanced stereoselectivity on increasing the catalyst loading (Table 1, entries 7-8). When the loading of organocatalyst 3 was increased to $12 \mathrm{~mol} \%$, the an$t i$-aldol product was obtained with good enantioselectivity (74\% ee). The breakthrough was achieved when $15 \mathrm{~mol} \%$ of catalyst $\mathbf{3}$ was employed with triethylamine as the co-catalyst.

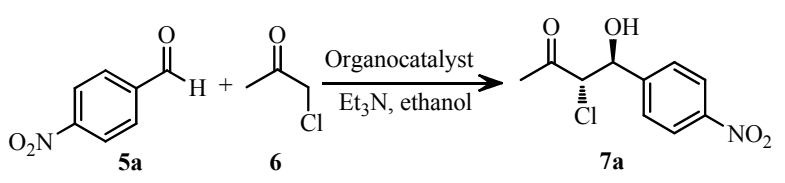

Scheme 1. Aldol reaction of chloroacetone with $p$-nitrobenzaldehyde.

Table 1

Optimization of the aldol reaction between chloroacetone and $p$-nitro benzaldehyde.

\begin{tabular}{lcccccc}
\hline Entry & Catalyst & $\begin{array}{c}\text { Catalyst } \\
(\mathrm{mol} \%)\end{array}$ & $\begin{array}{c}\text { Time } \\
(\mathrm{h})\end{array}$ & $\begin{array}{c}\text { Yield a }^{\mathrm{a}} \\
(\%)\end{array}$ & anti/syn $^{\mathrm{b}}$ & $\begin{array}{c}\text { anti ee }^{\mathrm{c}} \\
(\%)\end{array}$ \\
\hline 1 & $\mathbf{1}$ & 10 & 48 & 29 & $\mathrm{Nd}^{\mathrm{d}}$ & $\mathrm{Nd}^{\mathrm{d}}$ \\
2 & $\mathbf{2}$ & 10 & 48 & 40 & $2: 1$ & 36 \\
3 & $\mathbf{3}$ & 10 & 20 & 68 & $7: 1$ & 69 \\
4 & $\mathbf{4}$ & 10 & 24 & 59 & $5: 1$ & 57 \\
5 & $\mathbf{3}$ & 12 & 18 & 75 & $7: 1$ & 74 \\
6 & $\mathbf{3}$ & 15 & 15 & 90 & $8: 1$ & 79 \\
7 & $\mathbf{3}$ & 20 & 26 & 53 & $3: 1$ & 45 \\
8 & $\mathbf{4}$ & 15 & 19 & 61 & $4: 1$ & 66 \\
\hline
\end{tabular}

a Isolated yield.

b Determined by ${ }^{1} \mathrm{H}$ NMR.

c Determined by chiral-phase HPLC analysis.

d Not determined. 
Table 2

Aldol reaction of chloroacetone with various aldehydes.

\begin{tabular}{|c|c|c|c|c|c|c|}
\hline & $5 a-5 f$ & 6 & & & $7 \mathbf{a}-7 \mathrm{f}$ & \\
\hline Entry & $\mathrm{Ar}$ & $\begin{array}{l}\text { Time } \\
\text { (h) }\end{array}$ & Product & $\begin{array}{c}\text { Yield a } \\
(\%)\end{array}$ & anti/syn ${ }^{\mathrm{b}}$ & $\begin{array}{c}\text { anti ee }^{\mathrm{c}} \\
(\%)\end{array}$ \\
\hline 1 & $p-\mathrm{NO}_{2}-\mathrm{C}_{6} \mathrm{H}_{4}$ & 15 & $7 a$ & 90 & $8: 1$ & 92 \\
\hline 2 & $p-\mathrm{F}-\mathrm{C}_{6} \mathrm{H}_{4}$ & 15 & $7 b$ & 88 & $7: 1$ & 92 \\
\hline 3 & $p-\mathrm{CN}-\mathrm{C}_{6} \mathrm{H}_{4}$ & 16 & $7 c$ & 92 & $8: 1$ & 90 \\
\hline 4 & $p-\mathrm{Cl}-\mathrm{C}_{6} \mathrm{H}_{4}$ & 18 & 7d & 85 & $7: 1$ & 88 \\
\hline 5 & $o-\mathrm{Br}-\mathrm{C}_{6} \mathrm{H}_{4}$ & 20 & $7 e$ & 77 & $6: 1$ & 84 \\
\hline 6 & $\mathrm{C}_{6} \mathrm{H}_{5}$ & 24 & $7 f$ & 73 & $6: 1$ & 86 \\
\hline
\end{tabular}

${ }^{a}$ Isolated yield.

b Determined by ${ }^{1} \mathrm{H}$ NMR.

c Determined by chiral-phase HPLC analysis.

Up to $90 \%$ of the desired product 7 a was obtained with a higher selectivity (anti/syn was 8:1). The anti-aldol product was obtained with the excellent enantioselectivity of $92 \%$.

Further increase in the loading of catalyst $\mathbf{3}$ in the presence of triethylamine gave no more improvement, and the result showed significant deviation from the previous trend of optimization. The aldol reaction under the optimized condition using organocatalyst $\mathbf{4}$ in ethanol was also examined. This catalyst showed lower diastereoselectivity and enantioselctivity with decreased yield of the product 7a (Table 1, entries 5-7), even with an extended reaction time.

The scope of this class of aldol reactions using organocatalyst $\mathbf{3}$ and co-catalyst triethylamine in ethanol was examined with a series of arylaldehydes 5 (Table 2) and chloroacetones 6. Aromatic aldehydes with an electron-withdrawing substituent were excellent substrates. The reaction proceeded smoothly to afford the desired products $\mathbf{7 a - 7 f}$. In all cases, the reactions afforded the anti-aldol products in high yield with excellent enantioselectivity.

The scope of the catalyst system of chiral compound $\mathbf{3}$ and triethylamine was also assessed for the Knoevenagel condensation reaction between $p$-nitrobenzaldehyde $\mathbf{5 a}$ and ethylcyanoacetate $\mathbf{8}$ using ethanol as solvent (Scheme 2).

The base-mediated reaction at room temperature afforded the Knoevenagel adduct 9a in 69\% yield. A good increase in the product yield was observed with a slight raise in the temperature to $40^{\circ} \mathrm{C}$. At the still higher temperature condition of $50{ }^{\circ} \mathrm{C}$, the best yield for the Knoevenagel product was achieved. No significant change in yield was observed by a further increase in the temperature from $50{ }^{\circ} \mathrm{C}$ to $60{ }^{\circ} \mathrm{C}$. The influence of temperature on the Knoevenagel reaction is shown in Table 3.

Surprisingly, while experimenting on the same reaction at cold conditions, a byproduct was observed in very low percent yield. The byproduct was isolated by column chromatography

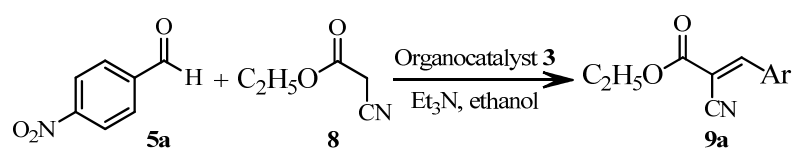

Scheme 2. Knovenagel reaction between $p$-nitrobenzaldehyde $\mathbf{5 a}$ and ethylcyanoacetate $\mathbf{8}$ using organocatalyst $\mathbf{3}$ and triethylamine.
Table 3

Knoevenagel reaction between ethylcyanoacetate and $p$-nitrobenzaldehyde at different temperatures in the presence of organocatalyst $\mathbf{3 b}$ (reaction time $20 \mathrm{~h}$ ).

\begin{tabular}{lccc}
\hline \multirow{2}{*}{ Entry } & \multirow{2}{*}{ Temperature $\left({ }^{\circ} \mathrm{C}\right)$} & \multicolumn{2}{c}{ Yield a ${ }^{\mathrm{a}}(\%)$} \\
\cline { 3 - 4 } & $\mathrm{RT}$ & $\mathbf{9 a}$ & $\mathbf{1 0 a}$ \\
\hline 1 & 40 & 69 & - \\
2 & 50 & 78 & - \\
3 & 60 & 89 & - \\
4 & 0 & 84 & - \\
5 & -20 & 54 & 26 \\
6 & -78 & 23 & 40 \\
7 & & 18 & 73 \\
\hline a Isolated yield. & & &
\end{tabular}

and analyzed for its structure. Our estimate was that the byproduct obtained could be 2-cyano-3-hydroxy-3-(4-nitrophenyl) propanoate formed during the reaction (Scheme 3). This was confirmed by the spectral data: proton NMR gave a broad singlet at $6.19 \mathrm{ppm}$ attributed to the presence of a hydroxyl group which was not found in the proton NMR of ethyl2-cyano-3-(4-nitrophenyl) acrylate. Further confirmation was provided by ${ }^{13} \mathrm{C}$ NMR which showed a signal at $74.83 \mathrm{ppm}$ for the carbon atom bearing the hydroxyl group. In the Knoevenagel product, the signal for the same carbon atom appeared at $151 \mathrm{ppm}$. The stability of product 10a decreased with increase in temperature.

The yield of product 10a was observed to increase with decrease in temperature. In the Knoevenagel reaction at a low temperature, 2-cyano-3-hydroxy-3-(4-nitrophenyl) propanoate is kinetically stable whereas at a higher temperature ethyl2-cyano-3-(4-nitrophenyl) acrylate is thermodynamically stable. After stirring for $20 \mathrm{~h}$, the reaction yielded $54 \%$ Knoevenagel adduct and $26 \%$ of product $10 \mathrm{a}$ at $0{ }^{\circ} \mathrm{C}$. At the lower temperature of $-20{ }^{\circ} \mathrm{C}$, only $23 \%$ of the Knoevenagel product 9a was observed with an increased yield of compound 10a. When the temperature was decreased to $-78{ }^{\circ} \mathrm{C}$, the percentage of kinetic stable product 10a was 73\%. However, at elevated temperatures, compound 2-cyano-3-hydroxy-3-(4nitrophenyl) propanoate was not observed. At these temperatures, only the Knoevenagel product was observed, which is thermodynamically more stable.

To check the necessity of the catalyst, the reaction was carried out in the absence of the catalyst. The reaction did not progress at a low temperature even after $48 \mathrm{~h}$ of stirring. However, at an increased temperature, the reaction showed some progress to give exclusively the Knoevenagel product. We also performed the reaction in the absence of the base triethylamine. The catalyst was able to promote the reaction to give a trace amount of product 9a only at high temperature. When the reaction was carried out with the replacing of ethyl-

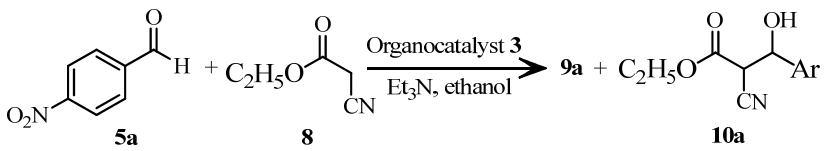

Scheme 3. Knoevenagel reaction between $p$-nitrobenzaldehyde $\mathbf{5 a}$ and ethylcyanoacetate $\mathbf{8}$ using organocatalyst $\mathbf{3}$ and triethylamine at low temperature. 
cyanoacetate by malononitrile or diethyl malonate, the reaction went to completion only at a high temperature to give only the Knoevenagel product.

We extended this protocol to other aldehydes to ensure the reproducibility of the results (Table 4). The reaction proceeded smoothly to give both the products with the hydroxy propanoate derivatives as the major product at $-78{ }^{\circ} \mathrm{C}$. When reaction was performed at room temperature, only the Knoevenagel product was obtained. The reaction was effective with electron withdrawing groups present on the aromatic ring while an electron donating substituent gave a moderate performance. Analysis by ${ }^{1} \mathrm{H}$ NMR spectra of the ethyl 2-cyano-3-hydroxy-3(aryl) propanoate derivatives showed that only the anti product was formed. We also determined the enantioselectivity to give the anti isomer of all products. The enantioselectivities were good to moderate.

Figure 2 shows a possible mechanism for the reaction, which explains the results. Triethylamine converts the keto form into the enol form by abstracting a hydrogen. The carbonyl group in $p$-nitrobenzaldehyde is activated by strong hydrogen bonding, which weakens the $\pi$ bond interaction between the carbon and oxygen of the carbonyl group and increases its electrophilicity. The enol attacks the $R e$-face of the carbon of the aldehyde, which leads to the formation of the favored transition state I shown in Fig. 2. The sulfur from the sulfonyl group contributes by the formation of van der Waals interaction, which plays a vital role in the transition state [32-36].

The hydroxy propanoate derivatives formed usually get dehydrated to give the Knoevenagel product. Due to the mild basicity, at low temperatures, the product does not get dehydrated, and hydroxy propanoate derivatives were obtained. However, at a higher temperature, the conditions were enough for the dehydration of the product formed in the presence of the base triethylamine.

Table 4

Aldol reaction of ethylcyanoacetate with various aldehydes.

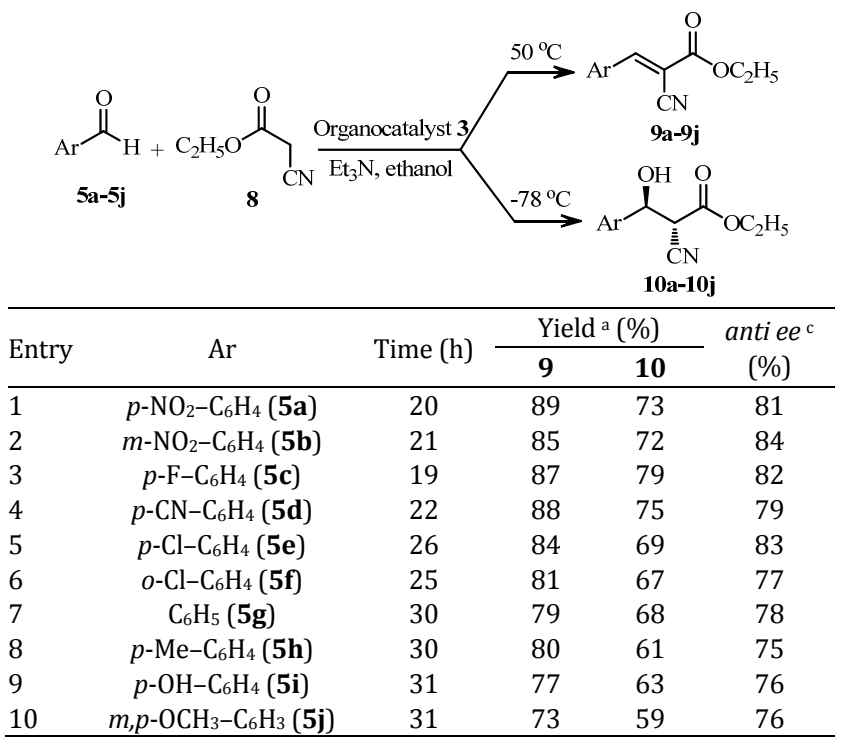

a Isolated yield at $50^{\circ} \mathrm{C}$.

b Isolated yield at $-78^{\circ} \mathrm{C}$

c Determined by chiral-phase HPLC analysis for compounds 10a-10j

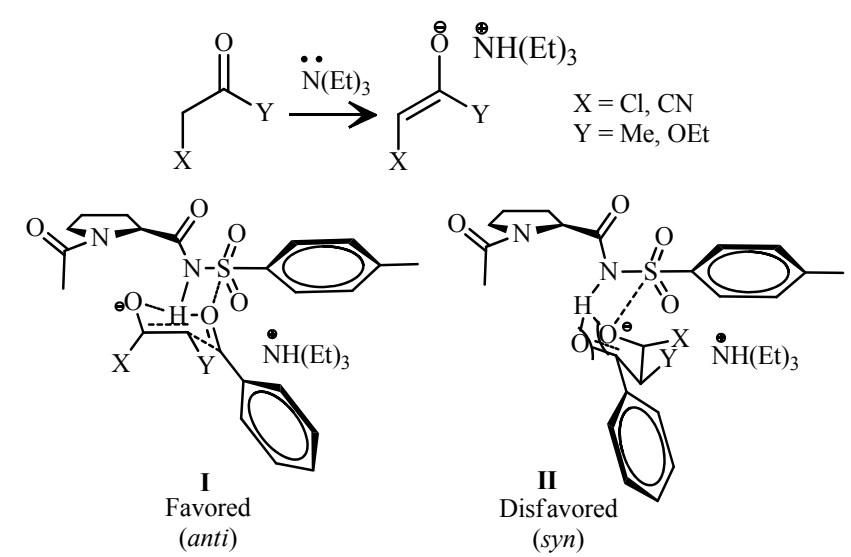

Fig. 2. Suggested transition state for the organocatalysed asymmetric aldol reaction.

\section{Conclusions}

We successfully used an organocatalyst in an asymmetric aldol reaction. Our hypothesis on hydrogen bonding catalysis using triethylamine and an organocatalyst having a pyrrolidine ring as the backbone was verified. The organocatalyst $(S)-1$ acetyl- $N$-tosylpyrrolidine-2-carboxamide $\mathbf{3}$ was an excellent catalyst. Extending the scope of our organocatalyst 3, we also studied the Knoevenagel reaction, which gave the usual Knoevenagel product at elevated temperatures and hydroxy propanoate derivatives at the low temperature of $-78{ }^{\circ} \mathrm{C}$. Our results also demonstrated that hydrogen bonding and van der Waals interactions are important in catalysis and can catalyze the reaction by stabilizing the intermediate. This protocol offers non-hazardous and eco-friendly reaction conditions with good yield and enantioselectivity.

\section{Acknowledgments}

We acknowledge Dr. P. L. More, Principal, Dnyanopasak College, Parbhani, for providing necessary facilities and IICT Hyderabad for providing spectral data.

\section{References}

[1] Lu C J, Luo Z H, Huang L, Li X S. Tetrahedron: Asymmetry, 2011, 22: 722

[2] Draper J A, Britton R. Org Lett, 2010, 12: 4034

[3] Yoshimitsu T, Nakatani R, Kobayashi A, Tanaka T. Org Lett, 2011, 13: 908

[4] Kang B, Britton R. Org Lett, 2007, 9: 5083

[5] Pajkert R, Kolomeitsev A A, Milewska M, Röschenthaler G V, Koroniak H. Tetrahedron Lett, 2008, 49: 6046

[6] Corey E J, Helal C J. Angew Chem Int Ed, 1998, 37: 1986

[7] Ohkuma T, Tsutsumi K, Utsumi N, Arai N, Noyori R, Murata K. Org Lett, 2007, 9: 255

[8] Knoevenagel E. Chem Ber, 1896, 29: 172

[9] Jones G. Org React, 1967, 15: 204

[10] Ramachary D B, Kishor M. J Org Chem, 2007, 72: 5056

[11] Ramachary D B, Ramakumar K, Narayana V V. J Org Chem, 2007, 72: 1458

[12] Kumar A, Maurya R A. Tetrahedron, 2007, 63: 1946 


\section{Graphical Abstract}

Chin. J. Catal., 2015, 36: 1093-1100 doi: 10.1016/S1872-2067(14)60317-X

\section{Stereoselective synthesis of vic-halohydrins and an unusual Knoevenagel product from an organocatalyzed aldol reaction: A non-enamine mode}

P. B. Thorat, S. V. Goswami, V. P. Sondankar, S. R. Bhusare*

Dnyanopasak College, India

Stereoselective synthesis of vic-halohydrins and an unusual Knoevenagel product were obtained using a newly synthesized chiral organocatalyst and triethylamine. The reaction gave the products in excellent yield with high anti selectivity and enantioselectivity.
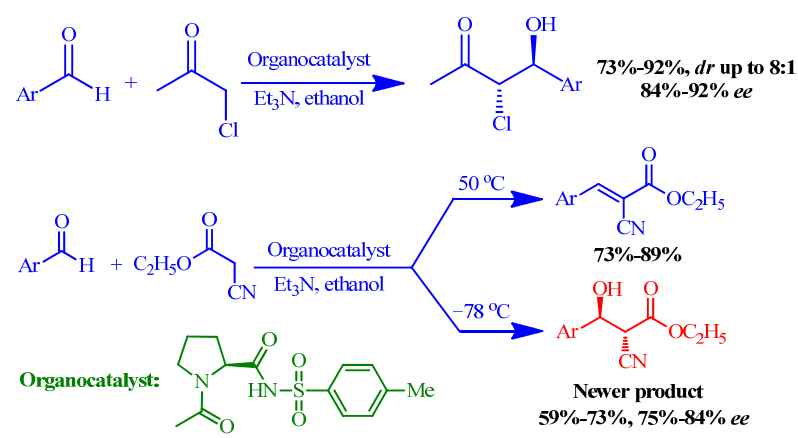

[13] Ramachary D B, Anebouselvy K, Chowdari N S, Barbas C F III. J Org Chem, 2004, 69: 5838

[14] List B, Lerner R A, Barbas C F III. J Am Chem Soc, 2000, 122: 2395

[15] Guillena G, Najera C, Ramon D J. Tetrahedron: Asymmetry, 2007, 18: 2249

[16] Seayad J, List B. Org Biomol Chem, 2005, 3: 719

[17] Dondoni A, Massi A. Angew Chem Int Ed, 2008, 47: 4638

[18] Ferreira F, Botuha C, Chemla F, Perez-Luna A. Chem Soc Rev, 2009, 38: 1162

[19] Morton D, Stockman R A. Tetrahedron, 2006, 62: 8869

[20] Robak M T, Herbage M A, Ellman J A. Chem Rev, 2010, 110: 3600

[21] Zhou P, Chen B C, Davis F A. Tetrahedron, 2004, 60: 8003

[22] Robak M T, Trincado M, Ellman J A. J Am Chem Soc, 2007, 129: 15110

[23] Thorat P B, Goswami S V, Khade B C, Bhusare S R. Tetrahedron: Asymmetry, 2012, 23: 1320

[24] Umehara A, Kanemitsu T, Nagata K, Itoh T. Synlett, 2012, 23: 453

[25] Liu Y, Liang J, Liu X H, Fan J C, Shang Z C. Chin Chem Lett, 2008, 19: 1043
[26] Sun Q, Shi L X, Ge Z M, Cheng T M, Li R T. Chin J Chem, 2005, 23: 745

[27] Yue C B, Mao A Q Wei Y Y, Li M J. Catal Commun, 2008, 9: 1571

[28] Yadav J S, Reddy B V S, Basak A K, Visali B, Narsaiah A V, Nagaiah K. Eur J Org Chem, 2004: 546

[29] Narsaiah A V, Nagaiah K. Synth Commun, 2003, 33: 3825

[30] Wang S, Ren Z, Cao W, Tong W. Synth Commun, 2001, 31: 673

[31] Lai Y F, Zheng H, Chai S J, Zhang P F, Chen X Z. Green Chem, 2010, 12: 1917

[32] Thorat P B, Goswami S V, Magar R L, Patil B R, Bhusare S R. Eur J Org Chem, 2013, 2013: 5509

[33] Thorat P B, Goswami S V, Jadhav W N, Bhusare S R. Aust J Chem, 2013, 66: 661

[34] Thorat P B, Goswami S V, Magar R L, Bhusare S R. J Chem Sci, 2013, 125: 1109

[35] Thorat P B, Goswami S V, Bhusare S R. Tetrahedron: Asymmetry, 2013, 24: 1324

[36] Thorat P B, Goswami S V, Khade B C, Bhusare S R. Tetrahedron Lett, 2012, 53: 6083 